\title{
P224: The influence of maximal barrier precaution on the prevention of central line-associated bloodstream infection in intensive care units
}

\author{
J Moon ${ }^{*}$, S Kim', J Im², J Baek², J Lee ${ }^{1,2}$ \\ From 2nd International Conference on Prevention and Infection Control (ICPIC 2013) \\ Geneva, Switzerland. 25-28 June 2013
}

\section{Introduction}

Although maximal barrier precaution (MBP) is recommended for the prevention of central line-associated bloodstream infection (CLABSI), use of MBP for the insertion of central venous catheter (CVC) in our hospital was very low.

\section{Objectives}

This study was intended to see whether improving the compliance for MBP during CVC insertion could reduce the incidence of CLABSI in the intensive care units (ICU).

\section{Methods}

This study was conducted at 5 intensive care units (total 53 beds, 2 medical ICUs, 1 cardiac ICU, 2 surgical ICUs) in one teaching hospital from Mar 2012 to Nov 2012. Infection control team educated proper practice on MBP during $\mathrm{CVC}$ insertion, monitored the CVC insertion procedure by using electronic check-list system, and provided feedback to physicians. The CDC-National Healthcare Safety Network definition for CLABSI was used. Chi-square test was done using Epi-info 6.0(Centers for Disease Control and Prevention, Atlanta, GA).

\section{Results}

MBP compliance rate during CVC insertion was 38.8\% before implementing this intervention. A total of 440 CVCs were undertaken for 9 months. Compliance rate was increased from average $47.8 \%$ of the first quarter (Mar May, 2012) to $79.6 \%$ of the third quarter(Sep Nov, 2012) $(p<0.001)$. The incidence of CLABSI was significantly improved from 4.00 (11/2,748 catheter days) per
1,000 catheter days during the first quarter to $0.70(2 / 2,817$ catheter days) per 1,000 catheter days $(p=0.011)$ at the third quarter.

\section{Conclusion}

MBP compliance for CVC insertion has increased through education, monitoring and feedback strategies with achieving the reduction of CLABSI.

\section{Disclosure of interest}

None declared.

\section{Author details}

${ }^{1}$ Department of Infection Control, Inha University Hospital, Korea, Republic Of. ${ }^{2}$ Division of Infectious Disease, Inha University, Incheon, Korea, Republic Of.

Published: 20 June 2013

doi:10.1186/2047-2994-2-S1-P224

Cite this article as: Moon et al.: P224: The influence of maximal barrier precaution on the prevention of central line-associated bloodstream infection in intensive care units. Antimicrobial Resistance and Infection Control 2013 2(Suppl 1):P224 